\title{
ASTROMETRIC STANDARD FIELDS FOR CCD OBSERVATIONS OF DOUBLE STARS
}

\author{
D. SINACHOPOULOS ${ }^{1}$, E. OBLAK ${ }^{2}$, M. GEFFERT ${ }^{3}$, J. COLIN $^{4}$, J.-F. \\ LeCAMPION ${ }^{4}$ and U. HOPP \\ ${ }^{1}$ Royal Belgian Observatory, Av. Circulaire 3, B-1180 Bruxelles, Belgium \\ ${ }^{2}$ Observatoire de Besançon, 41 bis, Avenue de L'Observatoire, F-25010 Besançon, \\ France \\ ${ }^{3}$ Sternwarte der Universität Bonn, W-5568 Daun, Germany \\ ${ }^{4}$ Observatoire de Bordeaux, F-33270, France \\ 5 Max-Planck-Institut für Astronomie, Königsstuhl 17, W-6900 Heidelberg 1, \\ Germany
}

\section{Photometry of Double Stars}

The number of known double stars is steadily increasing, thanks to ground-based and space observations. Therefore the ratio of known double to single stars has to be revised upwards continuously. With the Hipparcos parallaxes it will be possible to get stellar statistics for precisely defined volumes of space. This will lead to strong constraints on all astrophysical calibrations of masses, spectral types and luminosities in the solar neighbourhood. A European network of laboratories was created in August 1990 in order to remedy the lack of photometric data for close visual double stars. This network intends to study all aspects of formation and evolution of double and multiple star systems. The immediate goal of the group is to provide the scientific community with a compilation of known photometric data on a large selection of close visual double stars and to significantly enlarge this information by new observations with modern devices. A photometric database of stellar systems is being compiled from most widely used photometric systems in collaboration with the 'Centre de Donnees Stellaires' of Strasbourg.

\section{The Astrometric Calibration}

CCD frames of double stars normally have only small fields. Therefore an astrometric reduction with reference stars for the determination of position angle and separation of the components is not possible. Instead of that, the calibration has to be done by means of observations of test fields from which the scale and orientation of the frames may be determined. Instead of the standard double stars widely used recently, we have started to provide observers with astrometric standard fields. These fields are spread uniformly over a strip of the sky with $\alpha=0 \mathrm{~h}$ to $24 \mathrm{~h}$ and $\delta$ between $+20^{\circ}$ and $-20^{\circ}$. They contain 3 to 30 stars within a field of $2^{\prime} \times 3^{\prime}$ for a reliable calibration of the CCD-frames. Recently we have chosen eight fields. The data were taken partly from the literature or from measurement of new and old plates taken at the $(D=0.3 \mathrm{~m}$, 
$f=5.13 \mathrm{~m}$ ) photographic tube of the double refractor of Bonn (now located at Hoher List observatory). Only fields which have at least three bright stars $(\mathrm{V}<14)$ with precise positions $(\sigma<0.05)$ and proper motions $(\sigma<0.3 / 100 \mathrm{a})$ have been taken into account. Dense open cluster fields like the central field of M 67 (Girard et al. 1989) seem to be most suited, because they contain many well separated stars in a small field. The use of the outer parts of globular clusters is an alternative solution, although there may exist crowding problems. For the fields with low star density (3C 17, NGC 1647, 3C 273), we will provide two or three subfields with two or three calibration stars each.

Table 1. The astrometric test fields

\begin{tabular}{llll}
\hline Field & $\alpha_{2000}$ & $\delta_{2000}$ & Reference \\
\hline \hline 3C 17 & 00h 38m & $-02^{\circ} 07^{\prime}$ & New Measurements \\
NGC 1647 & $04 \mathrm{~h} \mathrm{45m}$ & $+19^{\circ} 07^{\prime}$ & Geffert et al. 1992 \\
NGC 2323 & $07 \mathrm{~h} \mathrm{42m}$ & $-14^{\circ} 49^{\prime}$ & New Measurements \\
M 67 & $08 \mathrm{~h} 50 \mathrm{~m}$ & $+11^{\circ} 49^{\prime}$ & Girard et al. 1989 \\
3C 273 & $12 \mathrm{~h} 28 \mathrm{~m}$ & $+02^{\circ} 03^{\prime}$ & Geffert et al. 1992 \\
M 12 & $16 \mathrm{~h} \mathrm{47m}$ & $-01^{\circ} 57^{\prime}$ & Geffert et al. 1992 \\
M 16 & $18 \mathrm{~h} \mathrm{19m}$ & $-13^{\circ} 47^{\prime}$ & Tucholke et al. 1986 \\
M 15 & $21 \mathrm{~h} \mathrm{30m}$ & $+12^{\circ} 10^{\prime}$ & New Measurements \\
\hline
\end{tabular}

\section{An Example: The $M 15$ Field}

M 15 is a globular cluster for which we have already published an astrometric catalogue (LeCampion et al. 1992). The central part of this catalogue was used to reduce measurements of four test plates taken with the $3.5 \mathrm{~m}$ telescope at Calar Alto in 1983 and scanned with the PDS in Münster. Our resulting catalogue has about 150 stars with a positional accuracy of 0.01 within a field of $15^{\prime} \times 15^{\prime}$, from which we may choose suited subfields. The final catalogue will be improved by $\mathrm{CCD}$ frames taken at the $60 \mathrm{~cm}$ telescope at the Observatoire de Bordeaux.

\section{Future Aspects}

We are planning to provide additional fields in other declination zones. The finding charts and coordinates of our fields are available on request.

\section{Acknowledgements}

We thank H.-J. Tucholke for scanning the Calar Alto plates. The financial support of the Ministère de la Recherche et de la Technologie, the Institut des Sciences de l'Univers, the Belgian Ministry of Research, and of travel funds from PROCOPE is gratefully acknowledged. 


\section{References}

Geffert, M., Sinachopoulos, D. and Guibert, J., 1992. IAU Coll. 135, 317.

Geffert, M., Tucholke, H.-J., Georgiev, Ts.B. and LeCampion, J.-F., 1991. Astron. Astrophys. Suppl., 91, 487.

Girard, T.M., Grundy, W.M., Lopez, C.E. and van Altena, W.F., 1989. Astron. J., 98, 227.

LeCampion, J.-F., Geffert, M., Dulou, M.R. and Colin, J., 1992. Astron. Astrophys. Suppl., 95, 233.

Tucholke, H.-J., Geffert, M. and The, P.S., 1986. Astron. Astrophys. Suppl., 66, 311. 